\title{
Origin and pulsation of hot subdwarfs
}

\author{
S. K. Randall ${ }^{1}$, G. Fontaine ${ }^{2}$, S. Charpinet ${ }^{3}$, V. Van Grootel $^{4}$, \\ and P. Brassard ${ }^{2}$ \\ ${ }^{1}$ ESO, Karl-Schwarzschild-Str. 2, 85748 Garching bei München, Germany \\ email: srandall@eso.org \\ ${ }^{2}$ Département de Physique, Université de Montréal, C.P. 6128, Succ. Centre-Ville, Montréal, \\ QC H3C 3J7, Canada \\ ${ }^{3}$ CNRS, Université de Toulouse, UPS-OMP, IRAP, 14 av. E. Belin, 31400, Toulouse, France \\ ${ }^{4}$ Institut d'Astrophysique et de Géophysique de l'Université de Liège, Allée du 6 Août 17, \\ B-4000 Liège, Belgium
}

\begin{abstract}
We briefly introduce hot subdwarfs and their evolutionary status before discussing the different types of known pulsators in more detail. Currently, at least six apparently distinct types of variable are known among hot subdwarfs, encompassing p- as well as g-mode pulsators and objects in the Galactic field as well as in globular clusters. Most of the oscillations detected can be explained in terms of an iron opacity mechanism, and quantitative asteroseismology has been very successful for some of the pulsators. In addition to helping constrain possible evolutionary scenarios, studies focussing on stellar pulsations have also been used to infer planets and characterize the rotation of the host star.
\end{abstract}

Keywords. stars: oscillations, subdwarfs

\section{Origin of hot subdwarfs}

Hot subdwarfs are compact, evolved stars located on the Extreme Horizontal Branch (EHB) of the Hertzsprung-Russell diagram. They are low-mass stars of about $0.5 M_{\odot}$ and comprise the spectral types sdB $\left(T_{\text {eff }} \sim 20000-36000 \mathrm{~K}\right)$ and $\mathrm{sdO}\left(T_{\text {eff }} \sim 36000-\right.$ $80000 \mathrm{~K}$ ). While most sdB stars are He-poor, the majority of sdO stars are He-rich. The chemical peculiarity observed in hot subdwarfs is caused by diffusion, the competitive action of radiative levitation and gravitational settling.

It is commonly accepted that hot subdwarfs are post red-giant stars that were stripped of too much of their H-envelope before or at the He-flash to sustain H-shell burning. In this canonical scenario, the sdB stars are in the core He-burning phase, while the $\mathrm{H}$ rich sdOs are their short-lived He-shell burning progeny already evolving away from the EHB (Dorman et al. 1993). He-rich subdwarfs on the other hand have been suggested to result from the merger of two He-rich white dwarfs (Saio \& Jeffery 2000) or a late He-core flash (Brown et al. 2001). One of the main problems in our understanding of the formation of hot subdwarfs is the question of what caused the necessary mass loss. Given that more than half of sdB stars in the field appear to reside in binary systems (e.g. Maxted et al. 2001), it has been proposed that the mass loss could have occurred as a consequence of binary formation channels involving stable Roche lobe overflow, a common envelope phase, or a combination of the two (Han et al. 2002, 2003). In this context, single sdB stars were suggested to have formed via the merger of two He white dwarfs, but this scenario was more recently superseded by the idea of a He white dwarf and main sequence star merger (Clausen \& Wade 2011). In globular clusters the EHB may have formed from a He-enriched sub-population (D'Antona et al. 2005). Another theory is that the mass loss was enhanced by the presence of planets (Soker 1998). 


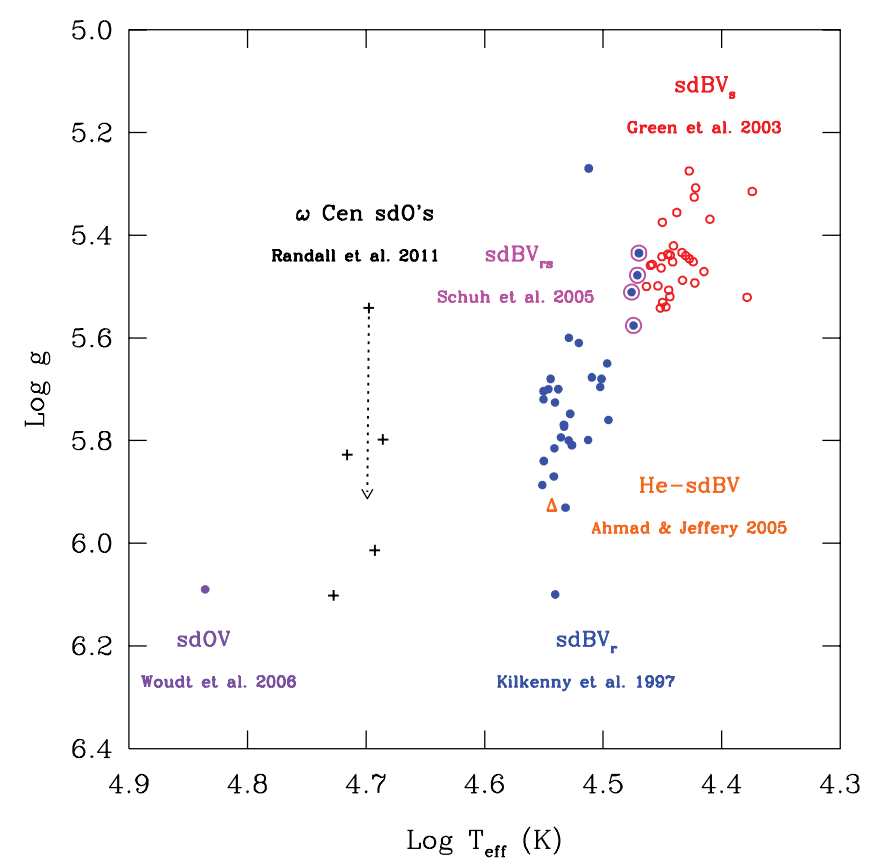

Figure 1. Currently known types of hot subdwarf pulsators. The references refer to the discovery paper of the prototype. The arrow pointing downwards for one of the $\omega$ Cen pulsators indicates that the $\log g$ value derived for this object is likely underestimated due to a significant contamination of its spectra by nearby stars.

The validity and relative importance of the proposed evolutionary scenarios as formation channels for the different populations of hot subdwarfs is still unclear. However, first trends are beginning to emerge based on observations of the binary properties of these stars, atmospheric abundance analyses and, more recently, the exploitation of pulsating hot subdwarfs through asteroseismology. The latter technique has the great advantage that not only the mass, but also the internal structure of the star can be determined to high accuracy. Moreover, pulsations can be used to infer substellar companions and planets, to test spin-orbit synchronization in binary systems and to characterise the internal rotation profile of the host star. In the following sections we describe the different types of known hot subdwarf pulsators and give a brief overview of our current understanding of these objects.

\section{Hot subdwarf pulsators}

The location of the different classes of subdwarf pulsators in the $\log g-T_{\text {eff }}$ diagram is displayed in Fig. 1. We now describe each class in more detail, working chronologically based on the time of their discovery.

\section{1. $s d B V_{\mathrm{r}}$ stars (also known as EC 14026 or V361 Hya stars)}

This first class of hot subdwarf pulsators was discovered (Kilkenny et al. 1997) independently, but almost at the same time as the theoretical prediction of such objects (Charpinet et al. 1996). Clustered in an instability strip between $\sim 29000$ and $36000 \mathrm{~K}$, $\mathrm{sdBV}_{\mathrm{r}}$ stars show short periods on a $100-300 \mathrm{~s}$ timescale that are interpreted as lowdegree, low-order p modes. The pulsations are driven by a $\kappa$-mechanism associated with a local overabundance of iron-peak elements in the driving region, which in turn is achieved 


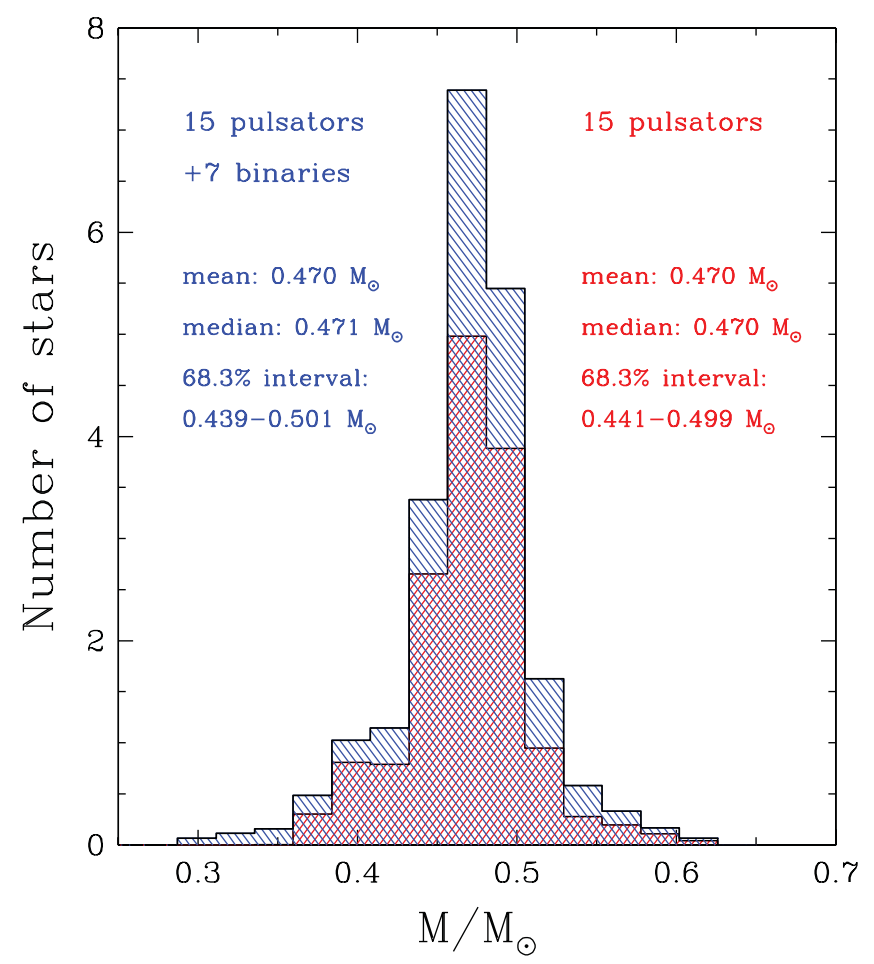

Figure 2. Empirical mass distribution based on the asteroseismological values for 15 sdBV stars as well as on estimates derived from the observed binary properties of 7 eclipsing binaries. The two distributions are indistinguishable from a statistical point of view.

by radiative levitation. Static envelope models incorporating the diffusion of traces of iron in a pure H-envelope (the so-called Montréal second-generation models, e.g. Charpinet et al. 1997) can very accurately reproduce the observed pulsation properties of these stars. Not only is the instability strip recovered almost perfectly (see Fig. 4), but quantitative asteroseismology via the forward method (e.g. Brassard et al. 2001) has also been very successful. For a discussion of the accuracy and precision achieved from the asteroseismology of $\mathrm{sdBV}_{\mathrm{r}}$ stars see Van Grootel et al., these proceedings.

To date, asteroseismic analyses have been carried out for 15 sdBV stars, allowing a first characterization of the mass distribution (Fig. 2; for more details see Fontaine et al. 2012). While we are still limited by small number statistics, there is tentative evidence that the high-mass $\left(\sim 0.5-0.7 M_{\odot}\right)$ tail arising primarily from the white dwarf merger scenario (Han et al. 2003) is much less pronounced than predicted. The empirical mass distribution peaks very close to the canonically expected value of $0.47 M_{\odot}$, and shows a similar behaviour to that predicted by the binary formation channels.

\section{2. $s d B V_{\mathrm{s}}$ stars (also known as V1093 Her or Betsy stars)}

The second type of pulsating hot subdwarf lies at the cool end of the sdB star regime $\left(T_{\text {eff }} \sim 22000-29000 \mathrm{~K}\right)$ and shows high radial-order $g$-mode oscillations on a timescale of 2000-8000 s (Green et al. 2003). While the driving mechanism was quickly identified to be the same iron opacity mechanism as for the $\mathrm{sdBV}_{\mathrm{r}}$ stars (Fontaine et al. 2003), standard models incorporating a non-uniform abundance profile only of iron are not able to reproduce the blue edge of the observed instability strip. A good agreement between 
the predicted and observed $\mathrm{sdBV}_{\mathrm{s}}$ instability regions was reached only recently using models including nickel as well as iron in the diffusion calculations (Hu et al. 2011).

Compared to the $\mathrm{sdBV}_{\mathrm{r}}$ stars, the slow pulsators are much more challenging to study, both from an observational and a modeling point of view. Ground-based observations are severely hampered by aliasing, atmospheric contamination and the length of the dataset needed, while the fact that g modes penetrate much deeper into the stellar interior compared to the shallower $\mathrm{p}$ modes means that the second-generation envelope models are no longer sufficient for quantitative modeling. Real progress on the asteroseismic exploitation of these objects was made only with the advent of space-bourne missions such as CoRoT and in particular Kepler, which delivered months of uninterrupted time-series photometry revealing typically hundreds of pulsation frequencies down to amplitudes of a few ppm. In parallel, progress was made on the computational front with the development of the Montréal third-generation models, which are complete static stellar models in thermal equilibrium that accurately describe both the stellar core and the envelope (for a detailed description see Van Grootel et al. 2013a). This has allowed quantitative asteroseismology to be carried out for a small number of $\mathrm{sdBV}_{\mathrm{s}}$ stars, and the mass and composition of the core to be constrained for the first time (Van Grootel et al. 2010a,b, Charpinet et al. 2011a).

Another interesting finding to come out of the Kepler data is that many of the g-mode pulsators also show a small number of very low-amplitude periodicities in the p-mode regime. Moreover, it appears that the $\mathrm{sdBV}_{\mathrm{s}}$ instability strip is not pure as was previously expected, but also contains a fraction $(\sim 25 \%$, based on small-number statistics $)$ of nonvariable stars. It is not yet clear why this is, or why the pulsator fraction is so much higher than for the $\mathrm{sdBV}_{\mathrm{r}}$ stars (where only $\sim 10 \%$ of stars in the instability strip pulsate). One explanation is that the apparently non-pulsating stars have not yet been able to build up the necessary reservoir of heavy elements in the driving region (Fontaine et al. 2006), but this remains to be investigated in more detail.

\section{3. $s d B V_{\mathrm{rs}}$ stars (also known as hybrid pulsators)}

These stars lie at the intersection of the $\mathrm{sdBV}_{\mathrm{s}}$ and $\mathrm{sdBV}_{\mathrm{r}}$ instability strips and exhibit both rapid p-mode and slow g-mode oscillations at appreciable amplitudes. First discovered by Schuh et al. (2005), a handful of such objects are now known. Hybrid pulsators are of great interest to asteroseismology, since both the inner and outer regions of the star can in principle be probed. A potential Rosetta stone, the hybrid pulsator and eclipsing binary 2M $1938+4603$ was recently observed with Kepler (Østensen et al. 2010). Asteroseismology however has so far been foiled, both by the very fast rotation of this object and the likely post-EHB status of this star.

\subsection{A He-sdBV:LSIV-14 116}

The unusual He-sdBV LS IV $-14^{\circ} 116$ (Ahmad \& Jeffery 2005) is located well within the $\mathrm{sdBV}_{\mathrm{r}}$ instability strip at $T_{\text {eff }} \sim 35000 \mathrm{~K}$ and $\log g \sim 5.9$, but quite puzzlingly exhibits g-mode pulsations in the same $(2000-5000 \mathrm{~s})$ period range as the $\mathrm{sdBV}_{\mathrm{s}}$ stars (Green et al. 2011). It is only moderately helium enhanced with $\log q(\mathrm{He} / \mathrm{H}) \sim-0.6$, but shows a strange abundance pattern with Ge, Sr, Y and Zr enhanced by factors of up to 10000 compared to solar values (Naslim et al. 2011). It has been proposed that these strange atmospheric abundances are indicative of a strong magnetic field, which may influence the driving of pulsation modes via the $\kappa$-mechanism and potentially shift the g-mode instability strip to higher temperatures. Alternatively, non-adiabatic pulsation calculations involving hot-flasher and post-merger evolutionary models suggest that periodicities on a similar time scale as those observed in LS IV $-14^{\circ} 116$ may be driven by the $\epsilon$-mechanism 


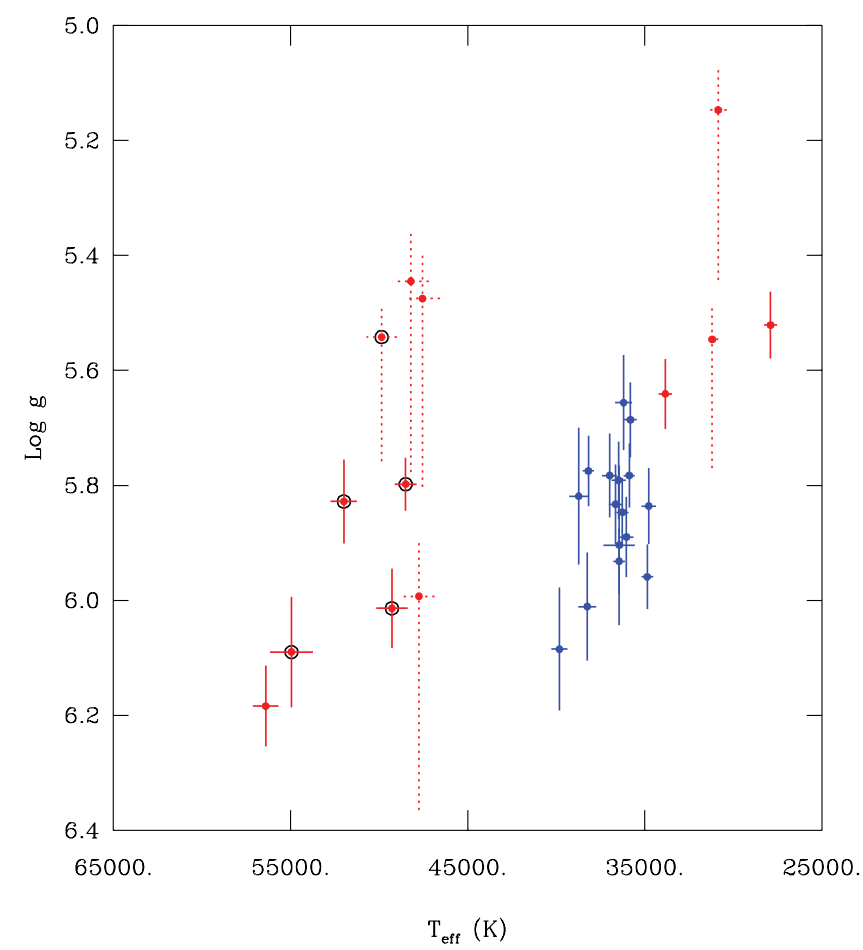

Figure 3. The instability strip in $\omega$ Cen. The five known pulsators are marked by large open circles, while the targets that were found to be constant down to a detection threshold of $<1 \%$ are denoted by small points. The continuous error bars refer to targets whose spectra were uncontaminated by nearby stars and for which reliable atmospheric parameters could be determined. Dotted error bars indicate stars with contaminated spectra, where in particular the derived $\log g$ value is likely to have been underestimated. The He-rich stars (blue in the online version) are clustered around $35000-40000 \mathrm{~K}$ while the remaining stars are H-rich.

acting on the unstable He-burning shells present just before the star settles on the EHB (Miller Bertolami et al. 2011, 2013).

2.5. An sdOV star among the field population: SDSS J160043.6 + 074802.9

To date the only confirmed sdO pulsator in the Galactic field is the very hot $(\sim 68500 \mathrm{~K})$, moderately helium-enhanced $(\log q(\mathrm{He} / \mathrm{H}) \sim-0.64$, Latour et al. 2011) star SDSS J160043.6 + 074802.9, which exhibits very rapid p-mode pulsations with periods in the $60-120$ s range (Woudt et al. 2006). These pulsations can be driven by the same iron opacity mechanism as the pulsations in the $\mathrm{sdBV}_{\mathrm{r}}$ and $\mathrm{sdBV}_{\mathrm{s}}$ stars (Fontaine et al. 2008, see also Fig. 4).

\subsection{The $\omega$ Cen sdOV stars: the first EHB pulsators detected in a globular cluster}

Members of this most recently discovered class of EHB pulsator (Randall et al. 2011) have so far only been found in the unusual globular cluster $\omega$ Cen. The five currently known variables (Randall et al. 2013) appear to form a homogeneous class in terms of pulsation properties and atmospheric parameters. They are all H-rich sdO stars, all exhibit pulsation periods in the $80-125 \mathrm{~s}$ range, and all fall within a nicely defined $T_{\text {eff }} \sim 48000-54000 \mathrm{~K}$ empirical instability strip (see Fig. 3). It is not yet clear whether or not all $\omega$ Cen sdOs within this strip are pulsating. However, it is becoming increasingly likely that these stars do not exist among the field sdO population in a similar relative 




Figure 4. p-mode instability strip predicted by the Montréal second generation models. Each large grid point refers to a model showing unstable radial modes in the p-mode regime, while models indicated by small grid points do not exhibit instabilities. The observed $\mathrm{sdBV}_{\mathrm{r}}$ stars are denoted by big (blue in the online version) points superposed on the grid, and the sdO variables are shown by crosses. The five crosses at $\sim 50000 \mathrm{~K}$ refer to the $\omega$ Cen pulsators (the dotted error bar extension to lower values of $\log g$ for one of the stars implies that the latter is likely underestimated due to the significant contamination of the extracted spectrum), whereas the isolated cross at higher temperatures indicates the field sdOV SDSS J160043.6 + 074802.9.

proportion to the entire EHB star population. In fact, a dedicated study monitoring 36 sdO stars (Johnson et al. 2013) failed to uncover a single pulsator. However, there is one object, $\mathrm{PB} 8783$, traditionally accepted as an $\mathrm{sdBV}_{\mathrm{r}}$ star by the community, that may in fact turn out to be a field counterpart to the $\omega$ Cen pulsators (Østensen 2012). Due to the strong contamination of its optical spectrum by an F-type main sequence companion there is a degeneracy when iteratively fitting the hot and the cool components during the atmospheric analysis, and both solutions giving a temperature of $\sim 36000 \mathrm{~K}$ (sdB) and $\sim 50000 \mathrm{~K}(\mathrm{sdO})$ are possible. An asteroseismic exploration of parameter space in the vicinity of the two spectroscopic solutions (Van Grootel et al. 2013b) indicated a preference for the hotter of the two. However, UV spectra will be needed in order to confirm the true nature of this rapid variable.

With the aim of understanding the non-adiabatic properties of the $\omega$ Cen variables, we extended the grid of Montréal second-generation models in order to cover the required region of $T_{\text {eff }}-\log g$ parameter space. The results of our instability calculations for lowdegree p-mode pulsations for hot subdwarfs between 20000 and $78000 \mathrm{~K}$ can be seen in Fig. 4. It becomes apparent that the $\mathrm{sdOV}$ and $\mathrm{sdBV}_{\mathrm{r}}$ star instability strips are in fact connected at low surface gravities. However, while the observed $\mathrm{sdBV}_{\mathrm{r}}$ instability strip is perfectly reproduced by the models, the $\omega$ Cen pulsators lie for the most part beyond the red edge of the theoretical sdO star instability region. Moreover, the periods excited by the models are too short compared to those observed. This could be due to shortcomings in the models (e.g. the fact that elements such as $\mathrm{Ni}$ are not incorporated in the opacity 
bump computations) or to problems with the accurate spectroscopic determination of the very high temperatures of these objects from optical spectra alone. Again, we need UV spectra to derive reliable temperatures for these objects.

\subsection{EHB pulsators in NGC 2808}

Recently, HST time-series photometry revealed 6 EHB pulsators in the central regions of the globular cluster NGC 2808 (Brown et al. 2013). While the intent of these observations had been to uncover counterparts to the $\omega$ Cen sdOVs in another globular cluster, the variables that were finally uncovered appear to be a rather inhomogeneous bunch, and none match the $\omega$ Cen oscillators in terms of atmospheric parameters and pulsation properties. The one NGC 2808 pulsator that has $T_{\text {eff }} \sim 50000 \mathrm{~K}$ is extremely He-rich and has a periodicity of $147 \mathrm{~s}$, longer than any of the $\omega$ Cen pulsators. The other two variables for which UV spectroscopy is available lie in the $\mathrm{sdBV}_{\mathrm{r}}$ and $\mathrm{sdBV} \mathrm{V}_{\mathrm{s}}$ regimes respectively, but both show very short periodicities around $110 \mathrm{~s}$. These findings are extremely puzzling, and present a significant challenge to our understanding of hot subdwarfs.

\section{Nice "side-effects" of pulsation studies}

The pulsations in hot subdwarfs have been used not only to determine the fundamental parameters of the stars themselves, but also to detect planets orbiting them. For several $\mathrm{sdBV}_{\mathrm{r}}$ stars that have been monitored extensively over several years, small sinusoidal frequency variations of the dominant pulsation modes have been measured and attributed to an orbital wobble caused by sub-stellar companions, either brown dwarfs or giant planets (Silvotti et al. 2007, Lutz et al. 2012). Two smaller, likely Earth-sized planets were inferred from extremely low-amplitude, low-frequency luminosity modulations detected


(or at least their cores) must have somehow survived the red giant stage, and perhaps they played a role in the formation of their host EHB star by enhancing the mass loss.

Another benefit of pulsations is that the rotation rate of the host star can be constrained by the measurement (or absence of) rotational splitting. While most single sdB stars are known to be slow rotators, the situation is different for those stars residing in close binaries. Depending on the proximity and nature of the companion, the rotation rate of the individual binary components will become synchronized with the orbital period. For example, in the 0.57-d $\mathrm{sdBV}_{\mathrm{s}}+$ white dwarf binary PG $0101+039$, a tiny ellipsoidal variation detected at exactly half the orbital period was used to confirm spinorbit synchronization (Geier et al. 2008). On the other hand, the rotational splitting of pulsation frequencies measured by Kepler in two $\mathrm{sdBV}_{\mathrm{s}}+\mathrm{dM}$ binaries with binary periods $\sim 0.4 \mathrm{~d}$ indicates that the rotation period is much longer $(\sim 7-10$ days $)$ than the binary period (Pablo et al. 2012). With asteroseismology it is possible to go even further and measure not just the rotation at the stellar surface, but also in the interior. To date, such measurements are consistent with solid-body rotation (e.g. Charpinet et al. 2008).

\section{References}

Ahmad, A. \& Jeffery, C. S. 2005, A\& A, 437, L51

Brassard, P., Fontaine, G., Billères, M., et al. 2001, ApJ, 563, 1013

Brown, T. M., Sweigart, A. V., Lanz, T., Landsman, W. B., \& Hubeny, I. 2001, ApJ, 562, 368

Brown, T. M., Landsman, W. B., Randall, S. K., Sveigart, A. V., \& Lanz, T. 2013, ApJ, 777, L22

Charpinet, S., Fontaine, G., Brassard, P., \& Dorman, B. 1996, ApJ, 471, L103 
Charpinet, S., Fontaine, G., Brassard, P., et al. 1997, ApJ, 483, L123

Charpinet, S., Van Grootel, V., Reese, D., et al. 2008, A\&A, 489, 377

Charpinet, S., Van Grootel, V., Fontaine, G., et al. 2011a, A\&SA, 530, A3

Charpinet, S., Fontaine, G., Brassard, P., et al. 2011b, Nature, 480, 496

Clausen, D. \& Wade, R. A. 2011, ApJ, 733, L42

D'Antona, F., Bellazzini, M., Caloi, V., et al. 2005, ApJ, 631, 868

Dorman, B., Rood, R. T., \& O'Connell, R. W. 1993, ApJ, 419, 596

Fontaine, G., Brassard, P., Charpinet, S., et al. 2003, ApJ, 597, 518

Fontaine, G., Brassard, P., Charpinet, S., \& Chayer, P. 2006, MemSAIt, 77, 49

Fontaine, G., Brassard, P., Green, E. M., et al. 2008, A\&SA, 486, L39

Fontaine, G., Brassard, P., Charpinet, S., et al. 2012, A\&A, 539, A12

Geier, S., Nesslinger, S., Heber, U., et al. 2008, A\&A, 477, L13

Green, E. M., Fontaine, G., Reed, M. D., et al. 2003, ApJ, 583, L31

Green, E. M., Guvenen, B., O'Malley, C. J., et al. 2011, ApJ, 734, 59

Han, Z., Podsiadlowski, P., Maxted, P. F. L., Marsh, T. R., \& Ivanova, N. 2002, MNRAS, 336, 449

Han, Z., Podsiadlowski, P., Maxted, P. F. L., \& Marsh, T. R. 2003, MNRAS, 341, 669

Hu, H., Tout, C. A., Glebbeek, E., \& Dupret, M.-A. 2011, MNRAS, 418, 195

Johnson, C. B., Green, E. M., Wallace, S., et al. 2013, arXiv: 1308.1373

Kilkenny, D., Koen, C., O'Donoghue, D., \& Stobie, R. S. 1997, MNRAS, 285, 640

Latour, M., Fontaine, G., Brassard, P., et al. 2011, ApJ, 733, 100

Lutz, R., Schuh, S., \& Silvotti, R. 2012, AN, 333, 1099

Maxted, P. F. L., Heber, U., Marsh, T. R., \& North, R. C. 2001, MNRAS, 326, 1391

Miller Bertolami, M. M., Córsico, A. H., \& Althaus, L. G. 2011, ApJ, 741, L3

Miller Bertolami, M. M., Córsico, A. H., Zhang, X., Althaus, L. G., \& Jeffery, C. S. 2013, in: J. Montalbán, A. Noels, \& V. Van Grootel (eds.), Ageing Low Mass Stars: From Red Giants to White Dwarfs, European Physical Journal Web of Conferences, 43, 4004

Naslim, N., Jeffery, C. S., Behara, N. T., \& Hibbert, A. 2011, MNRAS, 412, 363

Østensen, R. H. 2012, ASP-CS, 452, 233

Østensen, R. H., Silvotti, R., Charpinet, S., et al. 2010, MNRAS, 409, 1470

Pablo, H., Kawaler, S. D., Reed, M. D., et al. 2012, MNRAS, 422, 1343

Randall, S. K., Calamida, A., Fontaine, G., Bono, G., \& Brassard, P. 2011, ApJ, 737, L27

Randall, S. K., Calamida, A., Fontaine, G., et al. 2013, in: J. Montalbán, A. Noels, \& V. Van Grootel (eds.), Ageing Low Mass Stars: From Red Giants to White Dwarfs, European Physical Journal Web of Conferences, 43, 4006

Saio, H. \& Jeffery, C. S. 2000, MNRAS, 313, 671

Schuh, S., Huber, J., Green, E. M., et al. 2005, ASP-CS, 334, 530

Silvotti, R., Schuh, S., Janulis, R., et al. 2007, Nature, 449, 189

Soker, N. 1998, AJ, 116, 1308

Van Grootel, V., Charpinet, S., Fontaine, G., et al. 2010a, ApJ, 718, L97

Van Grootel, V., Charpinet, S., Fontaine, G., Green, E. M., \& Brassard, P. 2010b, A\&A, 524, A63

Van Grootel, V., Charpinet, S., Brassard, P., Fontaine, G., \& Green, E. M. 2013a, A\& $A$, 553, A97

Van Grootel, V., Charpinet, S., Fontaine, G., Brassard, P., \& Green, E. M. 2013b, ASP-CS, in press

Woudt, P. A., Kilkenny, D., Zietsman, E., et al. 2006, MNRAS, 371, 1497 